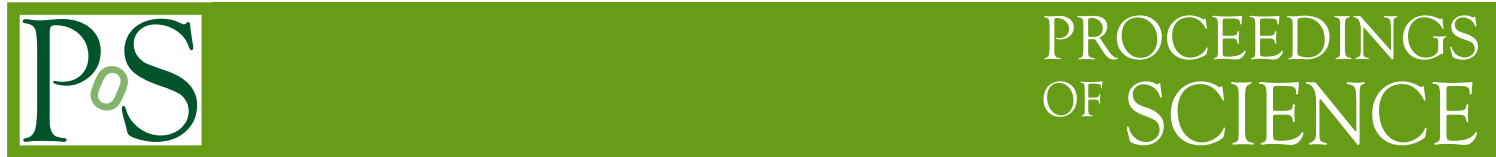

\title{
Dust lifetime re-evaluation in the light of a new dust model
}

\section{Bocchio* and A. P. Jones}

Institut d'Astrophysique Spatiale (IAS), UMR 8617, CNRS / Université Paris-Sud, 91405 Orsay,

France

E-mail: marco.bocchiodias.u-psud.fr

\begin{abstract}
We update an existing code that treats the destruction of dust in supernova-triggered shock waves, introducing more physics and new features in order to arrive at a more realistic modelling of dust processing in shock waves. We follow the evolution of the dust size distribution along the shock and calculate the dust emission after the shock. Finally, comparing the pre-shock and post-shock distributions, we estimate the integrated dust destruction and lifetime in our Galaxy. We find that both carbonaceous and silicate grain lifetimes are short compared to the timescale of their formation around evolved stars (stardust), thus affirming the long-standing conclusion that dust must be re-formed in the denser regions of the ISM.
\end{abstract}

The Life Cycle of Dust in the Universe: Observations, Theory, and Laboratory Experiments - LCDU 2013, 18-22 November 2013

Taipei, Taiwan

\footnotetext{
${ }^{*}$ Speaker.
} 


\section{Introduction}

It is now observationally well established that shock waves in the interstellar medium (ISM) are able to efficiently destroy dust grains [1,2]. Theoretical studies, e.g. [3, 4], estimated the dust destruction in supernova shock waves. They restricted their calculations to shocks in the warm ionised medium (WIM), which is where the dominant destruction occurs [5, 6]. As a result they found a lifetime of $\sim 2 \times 10^{8} \mathrm{yr}$ for carbonaceous grains and $\sim 4 \times 10^{8} \mathrm{yr}$ for silicate grains. On the other hand, the injection timescale from circumstellar regions has been estimated to be $\sim 3 \times 10^{9} \mathrm{yr}$ [7-9]. Clearly the injection timescale is much longer than the theoretical lifetime of dust and we would therefore expect to see a large fraction of heavy elements (i.e. $\mathrm{C}, \mathrm{Si}, \mathrm{Fe}, \mathrm{Mg}$, O, etc.) in the gas phase and almost nothing in dust grains. On the contrary, there is observational evidence that a large fraction of the heavy elements are locked into grains (i.e. $\geqslant 30 \%$ for $\mathrm{C}$ and $\geqslant 90 \%$ for $\mathrm{Si}, \mathrm{Fe}$ and $\mathrm{Mg}$ ) in the diffuse ISM [10]. This represents a long-standing conundrum and implies the reformation of dust grains by gas accretion in denser regions in the ISM in order to match the observed abundances in the diffuse ISM. It has been shown that there are viable mechanisms for the reformation of carbonaceous grains whilst experimental and observational results seem to indicate that silicate grains should survive from processing in the ISM [11].

In the following [12], we present an updated version of the existing GRASH code by [3, 4], where we introduce more physics and new features. Using this version of the code and a recent dust model [13] we follow the evolution of the dust size distribution in a shock wave and estimate the degree of dust destruction as a function of the shock velocity. Finally, we re-evaluate the dust lifetimes and compare them to the dust injection timescale.

\section{The GRASH_EX code}

The GRASH code is a FORTRAN code that follows the processing of dust in interstellar shock waves. The results of this code were first presented by [3] who implemented in the code the shock theory by [14] and the treatment of destructive processes [15]. The processes taken into account are thermal and inertial sputtering, vaporization and shattering of grains. In the original work the authors assumed graphitic and silicate grains following the MRN size distribution [16]. They did not solve simultaneously the shock dynamics and the grain physics but calculated the post-shock ionisation state self-consistently with the shock structure profiles.

In this study we update the original code (now called GRASH_EX) by introducing the following features:

- the carbonaceous material is updated from graphite to (hydrogenated) amorphous carbon, $\mathrm{a}-\mathrm{C}(\mathrm{H})$,

- the sputtering of the smallest carbonaceous grains is treated using a molecular approach $[17,18]$,

- thermal and inertial sputtering are merged into a single process,

- the charge scheme is updated from [14] to [19], 


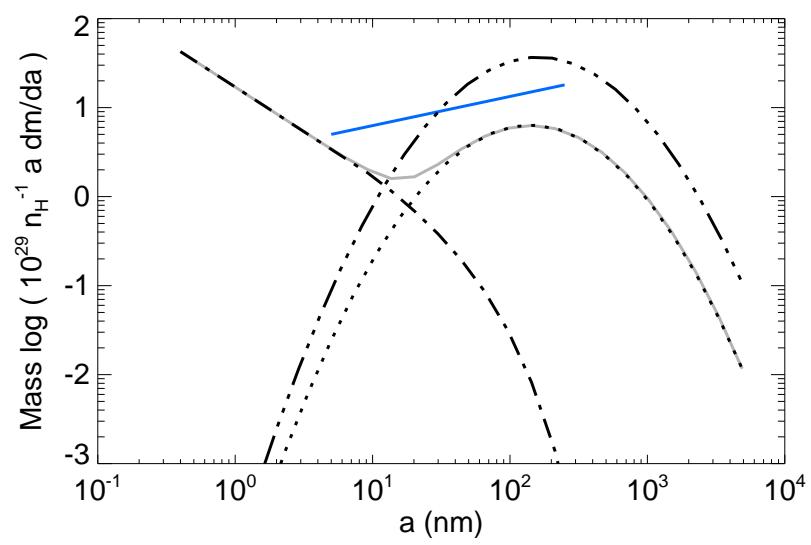

Figure 1: Size distribution of the three dust populations in the dust model [13]. The dot-dashed line and the dotted lines represents the small and large carbonaceous grain distributions, respectively, the sum of the two is represented by the grey solid line. The triple-dot-dashed line represents the silicate grain / carbon mantle size distribution. The blue solid line represents the standard MRN graphitic and silicate grain size distribution, as a comparison.

- we use a recent dust model [13] instead of the MRN dust model (see Fig. 1),

- the code is coupled to the DustEM code [20] (a code for the calculation of the dust emission and extinction), in order to estimate the dust emission in shocked regions.

\section{Results}

We assume a pre-shock density, $n_{0}$, and magnetic field, $B_{0}$, typical of the ionised regions in the warm interstellar medium (i.e. $n_{0}=0.25 \mathrm{~cm}^{-3}$ and $B_{0}=3 \mu \mathrm{G}$ ). We consider an initial ionisation fraction of $50 \%$ which corresponds to the precursor ionisation caused by the shock. We choose a shock velocity of $100 \mathrm{~km} \mathrm{~s}^{-1}$ for our "standard" run since it is close to the optimum of the shock frequency and destructive potential in interstellar shocks [14, 3, 4].

As a consequence of the grain processing during the shock, the carbonaceous mantles on the surfaces of the silicate grains are destroyed, leading to bare silicate grains. The outer aromatic layers of the carbonaceous grains [13] are partly destroyed as well but the typical shock timescale $\left(\sim 10^{5} \mathrm{yr}\right)$ and the sufficiently high radiation field $\left(G_{0}>1\right)$ lead to the re-aromatization of the outer $20 \mathrm{~nm}$ layer. Therefore, for all the shock velocities, the post-shock grains are characterized by bare silicate and carbonaceous grains with a $20 \mathrm{~nm}$ aromatic layer.

Fig. 2 shows the dust size distribution (left) and its spectral energy distribution (right) before and after the shock. In Fig. 2a, blue and orange lines refer to the initial size distribution of carbonaceous and silicate grains, respectively. Blue and orange boxes represent the two post shock size distributions. In Fig. $2 \mathrm{~b}$ the grey line represents the dust emission before the shock while the red line is the dust emission immediately after the shock. We notice that the size distribution is highly affected by the shock: small carbonaceous grains are mostly destroyed while the large 
silicate grains are highly affected by grain-grain collisions, i.e. the processes responsible for creating small silicate grains and leading to a broadening of the size distribution. These variations in the dust size distribution are reflected in its emission: the emission at short wavelengths is highly suppressed and the $10 \mu \mathrm{m}$ emission silicate feature becomes entangled with the a-C(:H) emission features (dashed line in Fig. 2b).

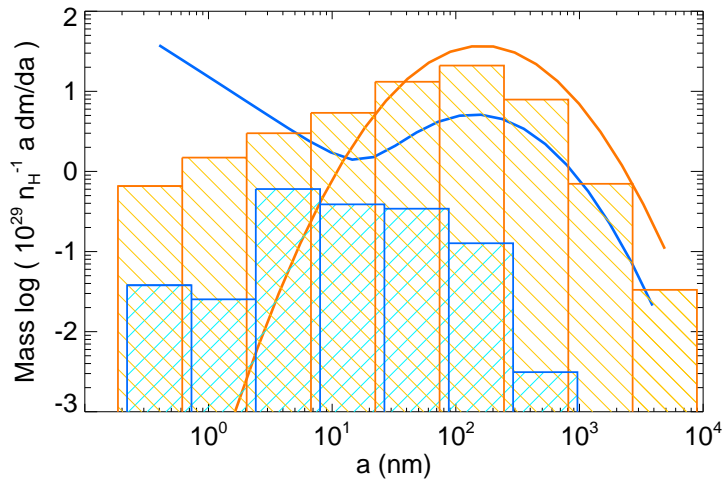

(a)

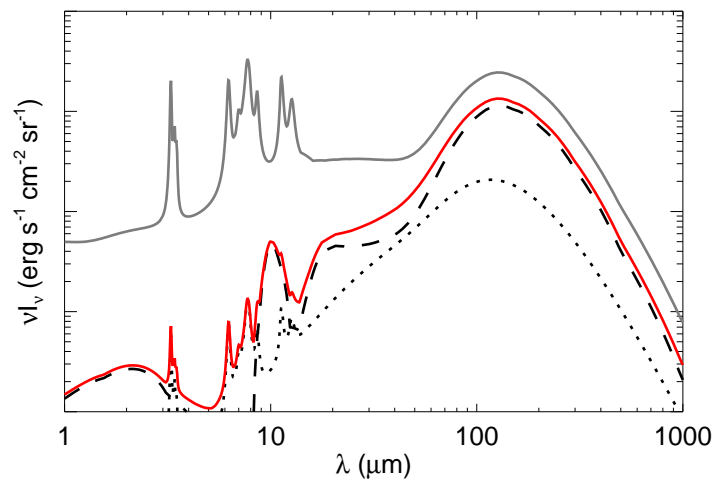

(b)

Figure 2: (a) Size distribution before and after a $100 \mathrm{~km} \mathrm{~s}^{-1}$ shock. (b) Dust SED before and after the shock.

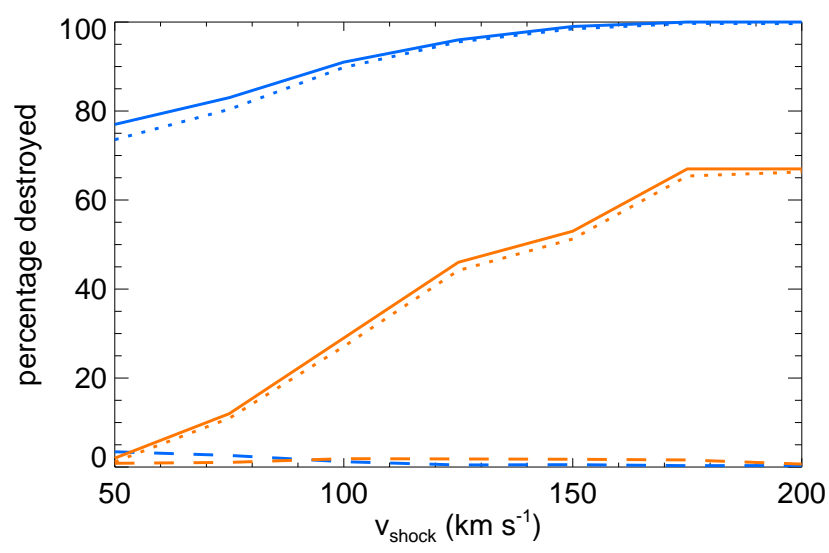

Figure 3: Dust destruction (\%) for carbonaceous grains (orange line) and silicate grains (blue line) as a function of the shock velocity. Dotted and dashed lines respectively represent the sputtering and vaporization contributions to the destruction.

Comparing the initial and final dust size distributions we then determine the percentage of carbonaceous and silicate grains destroyed during the shock. In Fig. 3 we plot the degree of destruction as a function of the shock velocity (same color-coding as per Fig. 2a). We notice that the erosion is dominated by the sputtering process (dotted lines) and that there is little contribution from vaporization (dashed lines). The destruction of carbonaceous grains is important even at 50 
$\mathrm{km} \mathrm{s}^{-1}$. This is due to the fact that in the dust model [13] almost $50 \%$ of the total carbonaceous mass is in small grains, which are quickly destroyed in a shock. On the other hand, silicate grains, being more resistant to sputtering, undergo less destruction.

\section{Astrophysical implications}

Following the approach by [6], with an estimation of the uncertainties as suggested by [11], we calculate the dust lifetime, $t_{\mathrm{SNR}}$, and the fraction of the elements locked up in stardust grains, $\delta_{\text {eq }}$. We can approximate the carbonaceous destruction efficiency as:

$$
\varepsilon\left(v_{s 7}\right)= \begin{cases}0.66+0.23 v_{\mathrm{s} 7} & \text { for } 0.5<v_{s 7} \leqslant 1.5 \\ 1 & \text { for } 1.5<v_{\mathrm{s} 7}<2.0\end{cases}
$$

and for silicate grains:

$$
\varepsilon\left(v_{s 7}\right)= \begin{cases}0.61 v_{\mathrm{s} 7}-0.31 & \text { for } 0.5<v_{s 7} \leqslant 1.25 \\ 0.11+0.28 v_{\mathrm{s} 7} & \text { for } 1.25<v_{s 7} \leqslant 2\end{cases}
$$

where $v_{s 7}$ is the velocity in units of $100 \mathrm{~km} \mathrm{~s}^{-1}, \varepsilon\left(v_{s 7}\right)$ is the efficiency of grain destruction for a shock velocity $v_{s 7}$. This leads to a grains lifetime of:

$$
t_{\mathrm{SNR}}= \begin{cases}(6.2 \pm 5.6) \times 10^{7} \mathrm{yr} & \text { for carbonaceous grains } \\ (3.1 \pm 2.7) \times 10^{8} \mathrm{yr} & \text { for silicate grains }\end{cases}
$$

If we assume that $t_{\text {in }} \sim 3 \times 10^{9} \mathrm{yr}$ as estimated by [7], [8], [9], the dust destruction timescale results are all shorter than the timescale for its formation around evolved stars. The equilibrium fraction of elements locked in grains then results:

$$
\delta_{\mathrm{eq}}= \begin{cases}0.018 \pm 0.016 & \text { for carbonaceous grains } \\ 0.08 \pm 0.07 & \text { for silicate grains }\end{cases}
$$

The estimate of the carbonaceous grain lifetime is almost an order of magnitude shorter than previous estimates [4] which implies that only a small fraction of the carbon is locked into grains. This is in contrast with observations [10]. On the other hand, this work shows a similar lifetime for silicate grains than previous estimates. However, this estimate is still much shorter (by a factor of an order of magnitude) than the injection timescale. The discrepancy between the calculated lifetime and the estimated dust injection timescale can be explained with the reformation of the dust in the ISM. It has been shown that there are viable mechanisms for the reformation of carbonaceous grains while pre-solar grain analysis seem to indicate that silicate grains are little affected by destruction in the ISM, which is in contrast with our calculations.

\section{Conclusions}

We predict the evolution of dust in a shock using the new dust model of Jones et al. [13] and we find that small grains are significantly destroyed even for the lowest velocity shocks that we 
considered $\left(50 \mathrm{~km} \mathrm{~s}^{-1}\right)$. On the other hand, silicate grains are less destroyed because they are more resistant but the collision of small carbon grains with large silicate grains leads to fragmentation of the large silicate grains and to a broadening of the size distribution. However, the dust lifetime is shorter than the injection timescale resulting in only a small fraction of the elements being locked into grains. This is in contrast with observations and implies that reformation of dust in the denser regions of the ISM must occur.

\section{References}

[1] Routly P. M. \& Spitzer L. Jr. 1952, ApJ 115, 227

[2] L. L. Cowie 1978, ApJ 225, 887-892

[3] Jones A. P., Tielens A. G. G. M., Hollenbach D. J. \& McKee C. F. 1994, ApJ 433, 797-810

[4] Jones A. P., Tielens A. G. G. M. \& Hollenbach D. J. 1996, ApJ 469, 740

[5] Seab C. G. 1987, Astrophysics and Space Science Library 134, 491-512

[6] McKee C. 1989, IAU Symposium, volume 135, page 431

[7] Dwek E. \& Scalo J. M. 1980, ApJ 239, 193-211

[8] Gehrz R. 1989, IAU Symposium page 445

[9] Jones A. P. \& Tielens A. G. G. M. 1994, pages 79-83

[10] Mathis J. S. 1990 ARA\&A 28, 37-70

[11] Jones A. P. \& Nuth J. A. 2011 A\&A 530, A44

[12] Bocchio M. \& Jones A. P. 2014, in prep.

[13] Jones A. P., Fanciullo L., Köhler M. et al. 2013, A\&A 558, A62

[14] McKee C. F., Hollenbach D. J., Seab G. C. \& Tielens A. G. G. M. 1987, ApJ 318, 674-701

[15] Tielens A. G. G. M., McKee C. F., Seab C. G. \& Hollenbach D. J. 1994, ApJ 431, 321-340

[16] Mathis J. S., Rumpl W. \& Nordsieck K. H. 1977, ApJ 217, 425-433

[17] Micelotta E. R., Jones A. P. \& Tielens A. G. G. M. 2010, A\&A, 510, A37

[18] Bocchio, M., Micelotta, E. R., Gautier, A.-L., \& Jones, A. P. 2012, A\&A, 545, A124

[19] Weingartner J. C., Draine B. T. \& Barr D. K. 2006, ApJ, 645, 1188

[20] Compiègne M., Verstraete L., Jones A. P. et al. 2011, A\&A 525, A103 